\title{
Evidence for the toxicity of bidirectional transcripts and mitochondrial dysfunction in blood associated with small CGG expansions in the FMR1 gene in patients with parkinsonism
}

\author{
Danuta Z. Loesch, MD, PhD ${ }^{1,2}$, David E. Godler, PhD', Andrew Evans, MD", Quang M. Bui, PhD ${ }^{4}$, \\ Freya Gehling, $B S c(H o n)^{5}$, Katya E. Kotschet, $M D^{6,7}$, Nicholas Trost, $M D^{7}$, Elsdon Storey, $M D, P h D^{8}$, \\ Paige Stimpson, PhD ${ }^{1,8}$, Glynda Kinsella, PhD ${ }^{1}$, David Francis, MSc ${ }^{2}$, David R. Thorburn, PhD ${ }^{9,10,}$ \\ Alison Venn, PhD ${ }^{11}$, Howard R. Slater, PhD ${ }^{2,10}$, and Malcolm Horne, MD, PhD 6,12
}

\begin{abstract}
Purpose: Our previous results showed that both gray zone and lower end premutation range ( $40-85$ repeats) fragile $\mathrm{X}$ mental retardation 1 (FMR1) alleles were more common among males with parkinsonism than in the general population. This study aimed to determine whether these alleles have a significant role in the manifestations and pathogenesis of parkinsonian disorders. Methods: Detailed clinical assessment and genetic testing were performed in 14 male carriers of premutation and gray zone FMRI alleles and in 24 noncarriers identified in a sample of males with parkinsonism. Results: The premutation + gray zone carriers presented with more severe symptoms than disease controls matched for age, diagnosis, disease duration, and treatment. The Parkinson disease (Unified Parkinson's Disease Rating Scale) motor score and the measures of cognitive decline (Mini-Mental State Examination and/or Addenbrooke's Cognitive Examination Final Revised Version A scores) were significantly correlated with the size of the CGG repeat and the (elevated) levels of antisense FMRI and Cytochrome C1 mRNAs in blood leukocytes. In addition, the carriers showed a significant depletion of the nicotinamide adenine dinucleotide, reduced dehydrogenase subunit 1 mitochondrial gene in whole blood. Conclusion: Small CGG expansion FMRI alleles (gray zone and lower end premutation) play a significant role in the development of the parkinsonian phenotype, possibly through the cytotoxic effect of elevated sense and/or antisense
\end{abstract}

From the ${ }^{1}$ School of Psychological Science, La Trobe University, Melbourne; ${ }^{2}$ Victorian Clinical Genetics Services Cytogenetics Laboratory, The Murdoch Childrens Research Institute, Royal Children's Hospital, Melbourne/Parkville; ${ }^{3}$ Department of Neurology, Royal Melbourne Hospital/Parkville; ${ }^{4}$ Centre for Molecular, Environmental, Genetic and Analytic, Epidemiology, University of Melbourne/Parkville; ${ }^{5}$ The Chromosome and Chromatin Research Laboratory, The Murdoch Childrens Research Institute, Royal Children's Hospital, Melbourne/Parkville; ${ }^{6}$ Centre for Clinical Neurosciences and Neurological Research, ${ }^{7}$ Department of Radiology, St Vincent's Hospital Melbourne/Fitzroy; ${ }^{8}$ Van Cleef Roet Centre for Nervous Diseases, Monash University, Melbourne; ${ }^{9}$ Mitochondrial and Metabolic Research Laboratory, The Murdoch Childrens Research Institute, Royal Children's Hospital, Melbourne/Parkville, Victoria, Australia; ${ }^{10}$ Department of Paediatrics, University of Melbourne/Parkville; ${ }^{11}$ Menzies Research Institute, University of Tasmania, Hobart, Australia; ${ }^{12}$ Florey Neurosciences Institute, Centre for Neuroscience, University of Melbourne/ Parkville, Victoria, Australia.

Dr. Danuta Z. Loesch, School of Psychological Science, La Trobe University, Victoria 3086, Australia. E-mail d.loesch@latrobe.edu.au.

Disclosure: The authors declare no conflict of interest.

Supplemental digital content is available for this article. Direct URL citations appear in the printed text and are provided in the HTML and PDF versions of this article on the journal's Web site (www.geneticsinmedicine.org).

Submitted for publication July 27, 2010

Accepted for publication November 10, 2010

Published online ahead of print January 25, 2011.

DOI: $10.1097 /$ GIM.0b013e3182064362
FMR1 transcripts involving mitochondrial dysfunction and leading to progressive neurodegeneration. Genet Med 2011:13(5):392-399.

Key Words: parkinsonism, premutation, gray zone, mitochondrial dysfunction, FMR1/ASFMR1/FMR4

Eragile X-associated tremor/ataxia syndrome (FXTAS), - OMIM\# 300623, first described in 2001, ${ }^{1}$ is a progressive late-onset neurodegenerative disorder associated with the premutation (PM) allele of the fragile $\mathrm{X}$ mental retardation 1 (FMRl) gene. ${ }^{2} \mathrm{PM}$ alleles contain small CGG repeat expansions in the promoter region ranging from 55 to $200 .^{3}$ Characteristic features of FXTAS are intention tremor, ataxia, and cognitive decline, ${ }^{1,4-6}$ with parkinsonian features occurring in approximately $57 \%$ of the affected males. ${ }^{4,5}$ Magnetic resonance imaging changes include white matter lesions of the middle cerebellar peduncles, combined with widespread cerebellar and cerebral white matter T2 hyperintensities. ${ }^{1,4-7}$ Neurohistological changes consist of ubiquitin-positive intranuclear inclusions throughout the brain. ${ }^{8}$

The prevalence of PM carriers in the general male population is approximately $1: 643,{ }^{9}$ and $40 \%$ of these carriers are likely to develop FXTAS as they get older. ${ }^{4,6}$ This has led to screening of patients with late-onset movement disorders for the presence of PM alleles. Although a higher prevalence of these alleles was found among males over 50 with cerebellar ataxia, OMIM\# 164400 or multiple system atrophy-cerebellar type, OMIM\# 146500 , it was not significantly increased in small samples of males with idiopathic Parkinson disease (iPD) or parkinsonism. ${ }^{10-13}$ In a larger study of 776 patients with iPD, three carriers of the PM allele were identified, ${ }^{14}$ which is approximately 3 -fold higher than the normal population prevalence.

The PM is associated with elevated levels of FMR1 mRNA, ${ }^{15}$ and the pathogenesis of FXTAS have been attributed to RNA becoming toxic through a "gain-of-function." $1,4,8,15$ Elevation of the FMR1 mRNA to the same "toxic" levels as in PM has also been reported in the carriers of gray zone (GZ) FMR1 alleles, ${ }^{16}$ where CGG expansions range from 45 to 54 repeats. ${ }^{3}$ It has been suggested that this finding may imply a possible role of these GZ alleles in the origin of the late-onset neurologic involvement already etiologically linked to the PM alleles. ${ }^{17}$ However, the role of these GZ alleles in the etiology of FXTAS is not clear because, unlike with PM alleles, the living carriers of GZ alleles do not give rise to, and therefore cannot be accessed through, probands affected with the Fragile $\mathrm{X}$ syndrome, OMIM\# 300624. Therefore, the prevalence and the phenotype of any GZ-related neurologic involvement cannot be determined in an unbiased manner. The alternative option is 
to screen those with disorders associated with tremor and/or ataxia for a possible excess of carriers of these alleles.

In contrast to the few earlier screening studies, ${ }^{12-14}$ we have previously reported a significant excess of both PM (10-fold increase) and GZ (2-fold increase) carriers among 228 Australian males diagnosed with typical idiopathic and atypical PD, OMIM\# 556500 (subsequently referred together as PD spectrum [PDS]), compared with the frequency in the general population. ${ }^{18}$

In this study, we present the results of in-depth molecular investigations of PM and GZ carriers identified in the PDS sample from this earlier study, ${ }^{18}$ comprising the levels of FMR1 mRNA and two mitochondrial measures: the extent of mitochondrial DNA (mtDNA) depletion and the levels of the Cytochrome $\mathrm{C} 1$ (CYC1) transcript, a nuclear oxidative-phosphorylation gene expressed during periods of mitochondrial expansion. ${ }^{19}$ We have also considered an antisense transcript (AFMR1, also known as FMR4), ${ }^{20}$ which has recently been identified within the FMR1 locus. Although its expression has been found to be elevated in the carriers of PM alleles, neither its levels in GZ allele carriers nor the relationship with clinical phenotype have as yet been investigated. The molecular measures have been compared between PDS carriers, PDS noncarriers, and healthy noncarriers and related to measures of severity of parkinsonian manifestations and cognitive decline across the whole range of repeat sizes. The results provide evidence that the size of the CGG repeat expansion, within both the GZ and the lower PM range, is a significant factor contributing to the origin and manifestations of PDS and that toxicity of the elevated FMR1 sense and antisense transcripts in both these allele categories may be linked to mitochondrial dysfunction.

\section{MATERIALS AND METHODS}

\section{Subjects}

Data collection and analysis were approved by the La Trobe University, St Vincent's Hospital, and Royal Melbourne Hospital Ethics Committees and by the Southern Tasmanian Health and Human Research Ethics and the University Ethics Committees at the Royal Hobart Hospital. Three carriers of PM (55-85 CGG repeats) and 11 carriers of GZ FMR1 alleles (40-54 CGG repeats) identified by screening in our earlier study of patients with PDS $^{18}$ were available, totaling 14 carriers of PM and GZ alleles aged from 54 to 82 years. All these individuals (later referred to as [PM + GZ]/PDS) furnished data on neurologic and neuropsychological scores and CGG repeat size; 12 of these individuals provided data on CYC1 and FMR1 transcript levels and 11 on ASFMR1 transcript levels. As transcriptional activity increases when FMR1 alleles exceed 39 repeats, ${ }^{16,18}$ we have here adopted 40 repeats as the lower boundary of the GZ range. Twenty-four subjects with normal size ( $<40$ CGGs) FMR1 alleles from the same sample of patients with PDS screened in our previous study ${ }^{18}$ and group matched for age and disease duration were selected as major disease controls (referred to as NS/PDS1). Most of these individuals provided neurologic and neuropsychological scores, and four also provided data on FMR1, ASFMR1, and CYC1 transcripts and mtDNA. These two groups, $(\mathrm{PM}+\mathrm{GZ}) / \mathrm{PDS}$ and normal size/PDS1, representing the range of CGG repeat sizes from normal, through GZ, to PM, were used in the analysis of relationships between molecular findings and clinical measures as shown in Table 1, and Figures 1 and 3.

A further sample of 20 PDS noncarriers, group matched for age (NS/PDS2), was also included to provide additional molec-

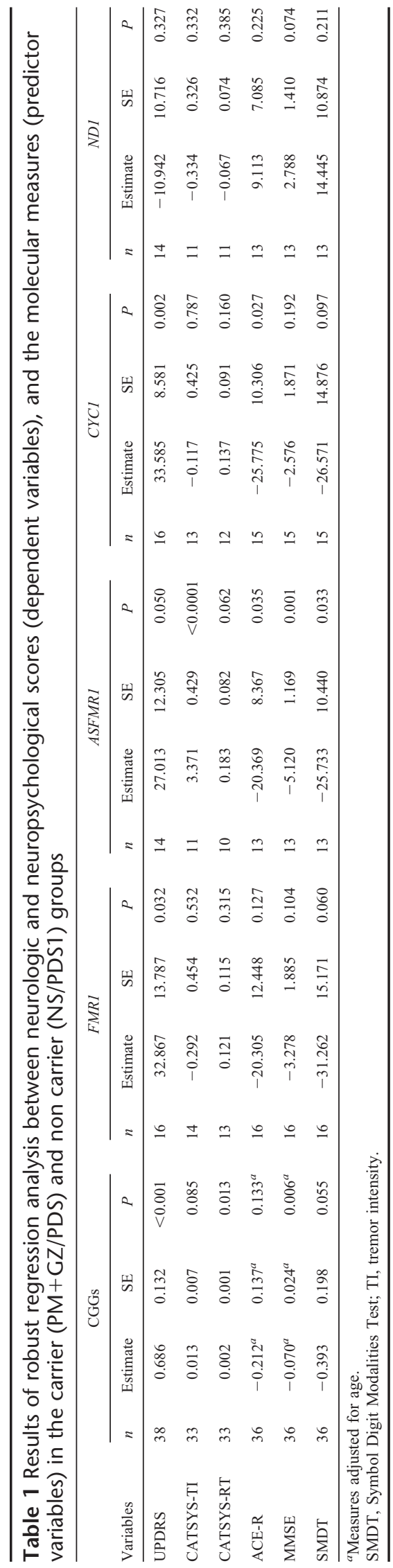


ular data for comparative analysis. As this group was not scored either for neurologic involvement or cognitive status, it was not included in the clinical-molecular relationship analysis. Ten of these individuals furnished data on FMR1 and ASFMR1 transcript levels and 13 on $\mathrm{CYC} 1$ transcript and nicotinamide adenine dinucleotide, reduced dehydrogenase subunit 1 (ND1) mtDNA depletion levels. Sixteen age-matched healthy controls (HCs) carrying normal size FMR1 alleles provided baseline data for FMR1 and CYC1 transcript levels and 12 of these, also for ASFMR1 mRNA and mtDNA levels. The data from all three PDS samples and $\mathrm{HC}$ samples were used to obtain the results shown in Figure 2. (The summary of data sources and sample sizes for each individual test used in the analyses is given in Table, Supplement Digital Content 1, http://links.lww.com/GIM/A137).

All patients with PDS from the carrier and noncarrier groups were receiving treatment with levodopa \pm dopaminergic agonists with a daily equivalent dosage of 7.8 (disease controls) and 10.7 (carriers), varying between individuals to achieve an optimal effect. One PM carrier of 56 repeats received deep brain stimulation to the subthalamic nucleus, in addition to the average levodopa dosage. The treatment duration was, on average, 1 year less than disease duration, which was 8.9 years (ranging 1-24 years) in the sample of carriers and 7.9 years (ranging $1-19$ years) in the sample of disease controls. All PDS subjects except one PM carrier of 85 CGG repeats had at least mild levodopa responsiveness. All participants were whites, mainly of northern European origin living in Australia.

\section{Laboratory techniques}

\section{CGG repeat sizing}

Processing of saliva samples and the assessment of the size of the CGG repeat from the extracted DNA (with a precision of 土one repeat) were conducted using a fully validated polymerase chain reaction (PCR) amplification assay, as detailed in our previous studies. ${ }^{21,22}$

\section{Blood sample processing}

Fifteen milliliters of blood was collected in ethylene diaminetetraacetic acid tubes (BD, worldwide) from all participants. DNA was extracted from $7.5 \mathrm{~mL}$ of whole blood using a BIO ROBOT M48 DNA Extractor (Qiagen Inc., Hilden, Germany). Peripheral blood mononuclear cells (PBMCs) were isolated from the remaining $7.5 \mathrm{~mL}$ of blood using Ficoll gradient separation, according to the manufacturer's instructions (Amersham Pharmacia Biotech, Uppsala, Sweden). Total RNA was extracted and purified from PBMCs using the RNeasy extraction kit, as per the manufacturer's instructions (Qiagen Inc., Hilden, Germany). The samples were stored at $-80^{\circ} \mathrm{C}$ until further analysis.

\section{$m t D N A$ depletion analysis using real-time $P C R$}

The 7900HT Fast Real-Time PCR machine (Applied Biosystems, Foster City, CA) was used to quantify the mtDNAencoded ND1 gene in DNA from whole blood. In this study, we modified the method originally developed for analysis of muscle and fibroblasts ${ }^{23}$ for whole blood, such that ND1 analysis was performed using an absolute real-time PCR standard curve method, ${ }^{24}$ normalized to total genomic DNA concentration determined using a NanoDrop ND-1000 Spectrophotometer. The ND1 dynamic linear range was performed on a series of doubling dilutions of a DNA standard (160-4 ng/ $\mu \mathrm{L})$ from whole blood. Previously published sequences were used for primers and for the probe for ND1,23 which were each used at a concentration of $10 \mu \mathrm{M}$. All samples were assayed in duplicate
$10 \mu \mathrm{L}$ PCR reactions. The reaction mixes consisted of $6.25 \mathrm{mM}$ $\mathrm{MgCl}_{2}, 1 \mu \mathrm{L}$ Buffer A (Applied Biosystems), 3.075 $\mu \mathrm{L}$ RNasefree water, $0.75 \mathrm{mM}$ deoxynucleotide triphosphates, 0.025 units/ $\mu \mathrm{L}$ of AmpliTaq Gold, $0.125 \mu \mathrm{L}$ of TaqMan probe and $0.25 \mu \mathrm{L}$ forward and $0.25 \mu \mathrm{L}$ reverse primers, and $2 \mu \mathrm{L}$ of DNA diluted to $2 \mathrm{ng} / \mu \mathrm{L}$. The annealing temperature for the thermal cycling protocol was $60^{\circ} \mathrm{C}$ for 40 cycles. The samples were quantified in arbitrary units in relation to the standard curves performed on each plate.

\section{$R N A$ extractions and reverse transcription real-time $P C R$}

Total RNA was extracted, purified, and reverse transcribed as described previously. ${ }^{21,25}$ The 7900HT Fast Real-Time PCR (Applied Biosystems) was used to quantify FMR1-5', FMR1-3', ASFMR1, GAPDH, B2M, and GUS, using the relative standard curve method. DNase treatment of total RNA was found to significantly affect levels of ASFMR1 and FMR1 (unpublished data) and, therefore, was not used for this study. The target gene and the internal control gene dynamic linear ranges were performed on a series of doubling dilutions of an RNA standard $(160-4 \mathrm{ng} / \mu \mathrm{L})$. A no reverse transcription enzyme control was included for every sample for ASFMR1 (as these assays did not target an exon/exon boundary). The difference between the plus and minus no reverse transcriptase control was considered as the ASFMR1 expression value for each sample. Previously published sequences were used for primers and probes for FMR1-5' and GUS ${ }^{15}$; FMR1-3'26; and ASFMR1 (FMR4). ${ }^{27}$ FMR1-5', FMR1-3', and ASFMR1 primers were used at a concentration of $18 \mu \mathrm{M}$ and probes at a concentration of $2 \mu \mathrm{M}$. EIF4A2 and SDHA primer/probe mixes were obtained from PrimerDesign (PerfectProbe ge-PP-12-hu kit) and used at a concentration of $2 \mu \mathrm{M}$. All the above assays were single plexed, with each sample assayed in duplicate $10 \mu \mathrm{L}$ PCR reactions. The reaction mixes consisted of $5.8 \mathrm{mM} \mathrm{MgCl}_{2}, 1 \mu \mathrm{L}$ Buffer A (Applied Biosystems), $3.35 \mu \mathrm{L}$ RNase-free water, $1.2 \mathrm{mM}$ deoxynucleotide triphosphates, 0.01 units $/ \mu \mathrm{L}$ of AmpliTaq Gold, $0.5 \mu \mathrm{L}$ of TaqMan probe and $0.5 \mu \mathrm{L}$ forward and $0.5 \mu \mathrm{L}$ reverse primers, and $1 \mu \mathrm{L}$ of the reverse transcription (cDNA) reaction. The annealing temperature for thermal cycling protocol was $60^{\circ} \mathrm{C}$ for 40 cycles. The samples were quantified in arbitrary units in relationship to the standard curves performed on each plate, standardized to the mean of the three internal control genes (GUS, EIF4A2, and $S D H A$ ).

\section{Clinical scores}

\section{Neurologic assessments}

A standard neurologic assessment was conducted using the Unified Parkinson's Disease Rating Scale (UPDRS) Part IIIMotor Examination. ${ }^{28}$ The (videotaped) assessments and scoring of both carrier and disease control samples were conducted by two neurology specialists (E.S. and K.E.K.), with relevant experience of the UPDRS, which has established interrater reliability. ${ }^{29}$ They were blind to the CGG results. Further quantitative assessment of tremor was conducted using the windowbased CATSYS 2000 system, Version 1.3.8 (Danish Product Development Ltd., DK-3070 Snekkersten, Denmark), ${ }^{30,31}$ which has been normalized using a sample size of 150 men and women, aged $20-70$ years. ${ }^{30}$ The measures considered were (a) postural tremor intensity measured by an accelerometer held like a pen in a horizontal plane approximately $2.5 \mathrm{~cm}$ from the umbilicus and (b) simple reaction time (RT), which measures a stimulus-response speed (in seconds) for sound signals given at random within fixed periods. 


\section{Neuropsychological measures}

Addenbrooke's Cognitive Examination Final Revised Version A (ACE-R) ${ }^{32}$ was used to screen for cognitive impairment. The total score (ACE-R full) was a raw score derived from the sum of the five subscores, measuring attention/orientation, memory, fluency, language, and visuospatial cognitive domains. A full Mini-Mental State Examination (MMSE) is embedded within the ACE-R and is particularly useful in the assessment of severity in patients with dementia, OMIM\# 127750. ${ }^{33}$ Processing speed was measured using the Symbol Digit Modalities Test. ${ }^{34}$ The testing was conducted by one trained neuropsychologist (P.S.) who was not aware of the participants' carrier status at the time of assessment.

\section{Statistical analyses}

The data were analyzed using STATA statistical software (Version 10.0, StataCorp, College Station, TX) and plotted using the publically available R package. ${ }^{35}$ Because of the small sample size and the non-Gaussian distributions, we compared the measures (including age, disease duration, UPDRS motor scores, FMR1 sense and antisense transcripts, CYC1 transcript, and levels of mtDNA) from the sample of carriers with those from disease controls and/or HCs, using the nonparametric Kruskal-Wallis test. Analysis of the relationships between major phenotypic and molecular measures was conducted using robust regression to downweight the effect of outliers. Both Huber weights and Tukey biweight, with the default biweight tuning constant of 7, were applied. We also applied least square estimations to assess the effect of outliers on the regression results.

Although the samples were group matched for age, both measures of dementia (MMSE and ACE-R) were found to be age related and were thus adjusted for age in all the regression analyses involving these two variables. However, there was no significant difference in disease (and thus treatment) duration between PDS carrier and noncarrier samples $(P=0.278)$, and this trait was not a significant confounder in any analyses conducted in this study.

\section{RESULTS}

We first tested the hypothesis that CGG expansion size and possibly the associated molecular changes are significantly re- lated to the severity of parkinsonian manifestations, including the level of cognitive decline. Strong evidence for the effect of CGG repeat size on these manifestations, based on data from 14 $(\mathrm{PM}+\mathrm{GZ}) / \mathrm{PDS}$ subjects and $24 \mathrm{NS} / \mathrm{PDS} 1$ controls, was provided by the finding (Fig. 1A) that the UPDRS motor score was significantly elevated in the sample of (PM + GZ)/PDS compared with NS/PDS1 $(P<0.001)$ and that this score was also significantly related to CGG repeat size (Fig. 1B). The CGG expansion size was also significantly correlated with the MMSE score (representing cognitive decline) and with RT assessed by CATSYS (Table 1). That the severity of neurologic involvement is also affected by other relevant molecular changes arising from small expansion FMR1 alleles is shown by the results of regression analyses that included the levels of both sense and antisense FMR1 transcripts as explanatory measures and several parkinsonian motor measures and cognitive scores as dependent variables (Table 1). Despite the small sample size, the levels of both these transcripts were significantly elevated in the (PM + GZ)/PDS sample compared with the NS/PDS1\&2 samples (Fig. $2, \mathrm{~A}$ and $\mathrm{B}$ ) and showed marginally significant associations with the UPDRS motor score. Notably, although FMR1 transcript levels were not significantly associated with other clinical measures, ASFMR1 levels showed significant correlation with all but one measure (CATSYS-RT).

We initially included CYC1 expression analysis to determine a set of the most stable reference genes for an assessment of the FMR1 mRNA levels from a set described by Vandesompele et al., ${ }^{36}$ using the geNorm algorithm. However, unlike any other reference genes included in the relevant kit (PrimerDesign kit PerfectProbe ge-PP-12-hu kit), the CYC1 gene showed differences in expression between the carrier and disease control groups (Fig. 2C). More specifically, the CYC1 transcript was found to be elevated in the (PM + GZ)/PDS sample relative to $\mathrm{HCs}$, and the levels of this transcript showed a highly significant relationship with the severity of parkinsonian manifestations (UPDRS motor score) and with cognitive decline (ACE-R) (Table 1 and Fig. 3A). Notably, CYC1 mRNA was also significantly increased in the NS/PDS1\&2 group compared with the HC subjects (two-sided $P=0.021$ ) but to a lesser extent than in the $(\mathrm{PM}+\mathrm{GZ}) / \mathrm{PDS}$ group (two-sided $P=0.01)$ (Fig. 2B). The highest levels of CYC1, as well as FMR1 and ASFMR1 transcripts in PBMCs, were found in two carriers with 85 and 170 CGG alleles, respectively (Fig. 2, A-D). These findings imply,
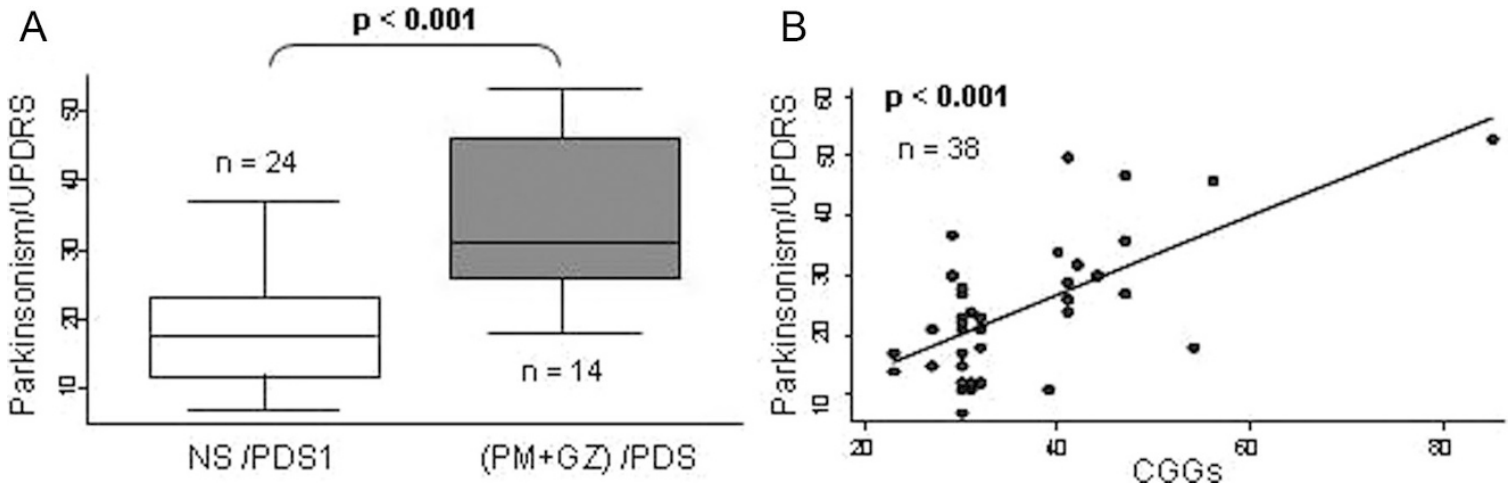

Fig. 1. Relationship between the UPDRS motor score and CGG expansion size based on data from a sample of carriers of PM and GZ alleles and a sample of disease controls, all identified through screening 228 Australian males affected with PDS. A, Boxplot showing the differences in UPDRS motor score between NS/PDS1 and PM + GZ/PDS samples. B, Scatterplots and regression lines fitted using robust regression of UPDRS motor score on CGG repeat size in the combined sample of PM and GZ carriers and disease controls. 

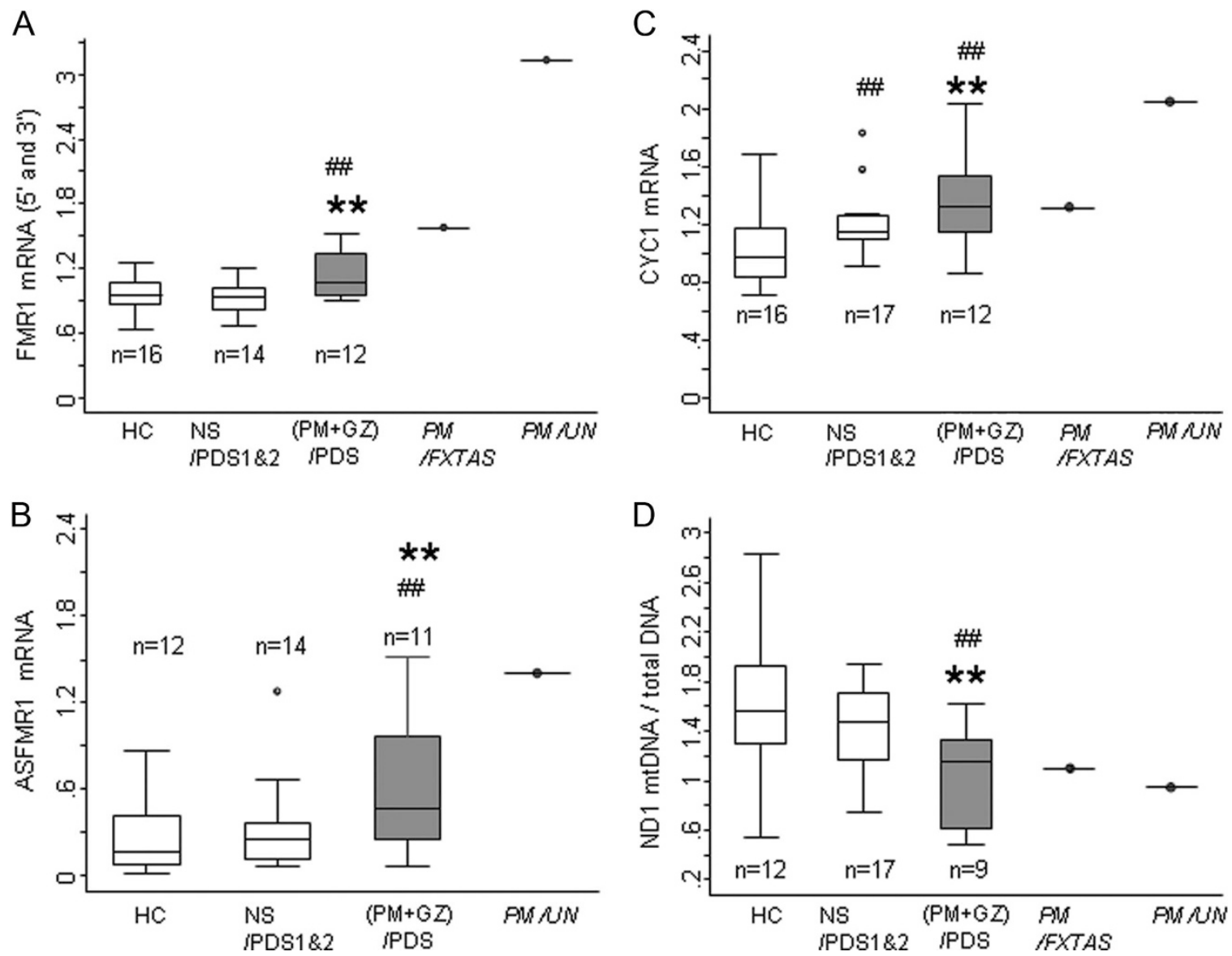

Fig. 2. Expression levels of sense (FMR1) and antisense (ASFMR1) transcripts, and a marker of mitochondrial dysfunction (ND1 mtDNA/total DNA) in peripheral blood of PM and GZ carriers (PM + GZ/PDS) compared with both disease control samples (NS/PDS1\&2), and healthy controls (HCs). A, FMR1 5'and 3'mRNA levels in PBMCs; B, ASFMR1 mRNA levels in PBMCs; C, CYC1 mRNA levels in PBMCs; and D, mtDNA ND1 levels in whole blood. PM/FXTAS is a PM carrier of 85 CGGs affected with FXTAS; and PM/UN is a PM carrier of 170 CGGs of unknown phenotype. Both these carriers were ascertained through fragile $X$ families and have not been included in global analyses. ${ }^{* *} P<0.05$ compared with $\mathrm{HCs}$; and \#\#P<0.05 compared with NS/PDS1\&2.

first, that the changes in CYC1 expression as seen in the (PM + GZ)/PDS sample may be relevant to the FMR1 mRNA toxicity, thus contributing to the severity of clinical manifestations of parkinsonism and second, considering that CYC1 expression levels are closely linked to the degree mitochondrial expansion or depletion, ${ }^{19}$ that mitochondrial dysfunction might be involved in toxicity of the FMR1 transcripts.

Therefore, as the next step, we assessed the levels of mtDNA in whole blood, where we found significantly reduced levels of ND1 gene mtDNA in the (PM + GZ)/PDS sample compared with both a subset of the NS/PDS1\&2 $(P=0.025)$ and $\mathrm{HC}(P=$ 0.019 ) groups (Fig. 2D). Despite the small samples sizes, ND1 mtDNA depletion was also significantly related to the number of CGG repeats within the PDS and GZ only category $(P=$ 0.011 ) (Fig. 3C) and to the rate of cognitive decline as represented by MMSE scores $(P<0.001)$ (Fig. 3D).

The scatterplots in Figure 3, A-D show that the significant relationships between phenotypic measures and the molecular changes have not been driven by single outliers in small samples but represent a general trend in the data. This has been confirmed using least square estimations, which showed that, if the outliers (numbering between 0 and 4 for individual correlations) were omitted from the analysis, or if there were no outliers (such as for correlations involving the UPDRS), the $P$ values were identical or almost identical to those obtained from the robust regression analysis. If the outliers were left in the analysis, the correlations became insignificant $(P>$ 0.05) for CATSYS-RT/CGG and ACE-R/ASFMR1 relationships but remained significant for the MMSE relationships with CGG repeat numbers and ASFMR1 transcript levels. Examples of the regression lines using both robust and least square methods are given in Figure, Supplemental Digital Content 2, http://links.lww.com/GIM/A138.

\section{DISCUSSION}

We have shown that the size of FMR1 CGG expansion within the low PM and GZ range affects the severity of parkinsonian manifestations in a sample of carriers of these alleles previously identified within a cohort of subjects diagnosed with PDS, ${ }^{18}$ irrespective of age and disease duration. The pattern of the relationships between the CGG repeat size and the severity 


Key: - PM/PDS • Normal size CGGs/PDS • GZ/PDS
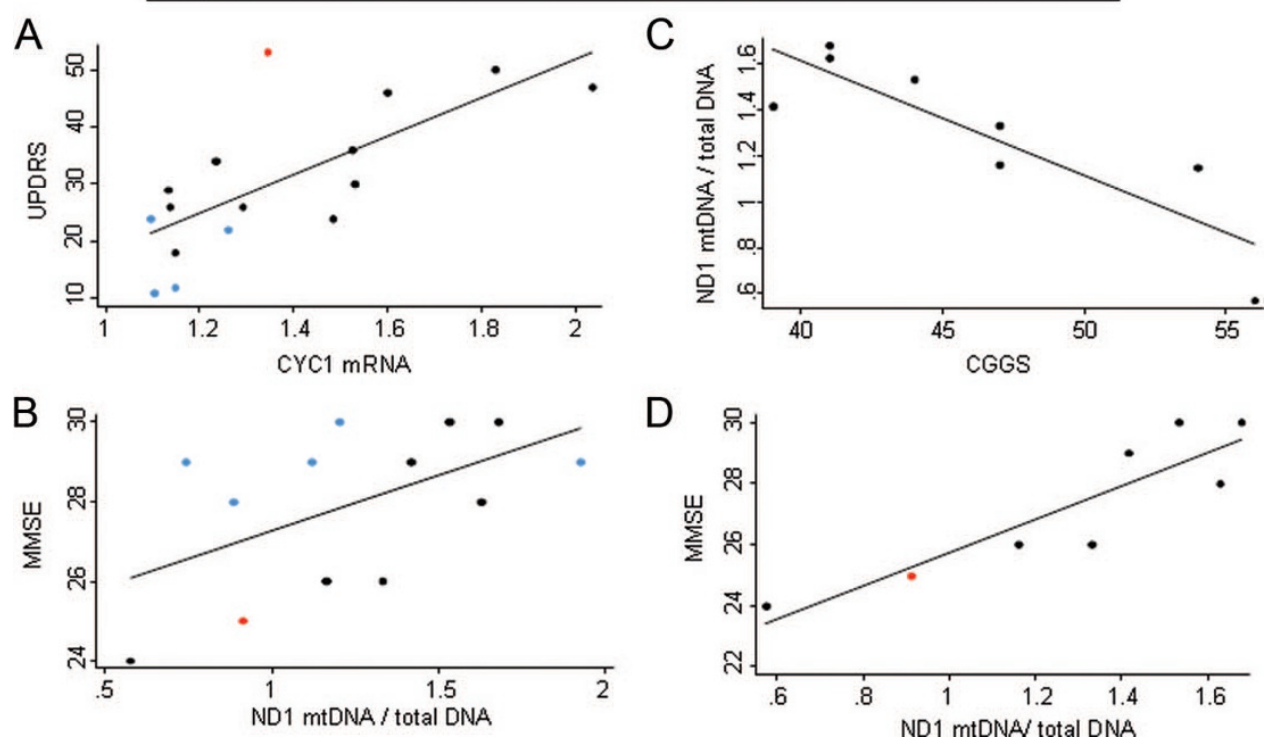

Fig. 3. Examples of relationships between the molecular measures in blood and neurologic and neuropsychological scores based on data from a sample of carriers of PM and GZ alleles and a sample of disease controls, all identified through screening 228 Australian males affected with PDS. Plots and fitted lines from robust regression of (A) UPDRS versus CYC1 mRNA; (B) MMSE versus ND1 mtDNA quantity in the combined sample; (C) CGG size versus ND1 mtDNA quantity in GZ carriers only; and (D) MMSE versus ND1 mtDNA quantity in GZ carriers and the one PM carrier.

of the motor phenotype and cognitive decline across the normal and $\mathrm{PM} / \mathrm{GZ}$ range suggest an incremental effect of the repeat size. The previously reported significant relationship between cognitive decline and the size of one CGG repeat across the whole range of small expansions and the normal range in a sample of patients with $\mathrm{PD}^{12}$ is consistent with our findings. These results together support our hypothesis from our earlier study ${ }^{18}$ that CGG repeat expansions encompassing both the GZ and the low-end PM range may contribute to phenotypic acceleration or modification of PD by synergizing with mechanisms relevant to its etiology and pathogenesis. Unlike larger alleles within the PM range, the allele sizes within the lower PM range and the GZ range may not be sufficient to produce FXTAS but may predispose the carriers to other conditions such as parkinsonism, where similar pathomechanisms may converge, resulting in increased risk or severity of manifestations of this disorder.

The major objective of this study was to uncover possible mechanisms involved in FMR1 mRNA toxicity and leading to neurodegenerative changes. A novel finding from this study is that the elevated levels of the sense (FMR1) and especially of the antisense (ASFMR1) transcripts also showed an incremental effect on the severity of the parkinsonian phenotype. The unanticipated finding of an effect of CYC1 expression on this phenotype, combined with the observed correlation of $\mathrm{CYC} 1$ expression levels with CGG repeat size and with the levels of both FMR1 transcripts, suggested that the effect of CGG expansions on the parkinsonian phenotype might be mediated through mitochondrial dysfunction. This is supported by the finding of significant mtDNA depletion in whole blood in the GZ/PM group compared with either HC or PDS noncarrier samples.

Evidence for mitochondrial dysfunction related to CGG expansions within the PM range has also been recently described in fibroblasts and brain material from patients with FXTAS ascertained through fragile $\mathrm{X}$ families. ${ }^{37}$ The results from that study are consistent with our findings of relationships between CGG expansion size, FMR1 and ASFMR1 transcript levels, and selected markers of mitochondrial dysfunction, with phenotypic measures. This suggests that the mitochondrial dysfunction related to RNA toxicity in accessible cell types such as fibroblasts and blood leukocytes, which have been used in our study, is likely to be paralleled by mitochondrial dysfunction in brain cells. Our findings, if confirmed in a larger sample, may contribute to a development of convenient molecular markers in blood, including CYC1 expression and mtDNA depletion, as useful predictors of disease severity and/or response to treatment. In our study, we have shown that mitochondrial dysfunction may also be associated with the much more common GZ alleles, thereby contributing to the severity of neurologic manifestations in patients with PDS carrying these alleles.

Another unprecedented finding of our study was that of an elevation of CYC1 levels in patients with PDS compared with $\mathrm{HCs}$, with a further increase in these levels in PDS GZ/PM carriers; a similar pattern was encountered for the ND1 mtDNA depletion. However, the interpretation of this finding is not straightforward. Because of the links of $\mathrm{CYC} 1$ with mitochondrial complex III, ${ }^{38}$ which is required to maintain functionality of complex I in mammalian mitochondria, ${ }^{39}$ the over or underexpression of CYC1 may potentially lead to destabilization of complex I. For example, mutations in the $C Y C 1$ gene have been reported to lead to a loss of functional mitochondrial complex III. Both impairment of complex I in substantia nigra and platelets and subsequent oxidative stress have been proposed as major factors in the pathogenesis of $\mathrm{PD},{ }^{40}$ and the interaction of this pathway with other potential factors causing oxidative stress has been postulated. ${ }^{41}$ In addition to lowered complex I activity in platelet mitochondria of patients with $\mathrm{PD}$, reduction 
in complex II/III activity (which may be relevant to aberrant CYC1 expression) has also been reported in this disorder. ${ }^{42}$

The elevation of CYC1 in PDS noncarriers that we have shown may reflect a specific pathway linked to complex I dysfunction or may be part of a broader cellular response, for example, secondary to loss of mtDNA. Mitochondrial dysfunction can result in up and downregulation of a range of specific nuclear genes, in an integrated stress response or retrograde response that varies in different cell types. ${ }^{43,44}$ The finding that CYC1 is further elevated in carriers raises the possibility that the observed mitochondrial changes in the GZ/PM carriers may interface with the oxidative stress pathway associated with PDS, leading to a more severe phenotype by exacerbating oxidative stress or dysfunction in mitochondrial pathways. This possibility is also suggested by earlier reports of elevated lactate levels in the lateral ventricles in both patients with FXTAS $^{45}$ and patients with iPD. 46

At this stage, it is not clear how the suggested mitochondrial dysfunction in carriers of PM/GZ alleles may be linked to the "toxicity" of elevated FMR1 transcript levels, but a deeper understanding of the disease mechanisms associated with these alleles may raise the possibility of treatment. It is possible that some proteins sequestrated through contact with the expanded FMR1 RNA ${ }^{47}$ have a physiological function in mitochondria and, through the "gain of function" mechanism previously postulated for alpha-synuclein toxicity, ${ }^{48}$ may cause dysfunction of these organelles.

In conclusion, our data, although based on small samples, show that the presence of either GZ or lower end PM alleles contributes significantly to the pathogenesis of parkinsonism, possibly through the cytotoxic effect of elevated FMR1 and/or ASFMR1 mRNA leading to mitochondrial dysfunction. Considering the exceptionally high prevalence of GZ alleles, which are carried by at least 1 in 30 individuals in the general population, ${ }^{9}$ larger studies are required to confirm these findings and also to establish whether this factor increases the risk for, and severity of, iPD, or determines a phenotypically overlapping entity within the heterogeneous group of primary parkinsonian disorders. Moreover, the link between the elevated FMR1 transcript and mitochondrial dysfunction, and the relevance of these to neuronal cell death, should be further explored through functional experiments. Furthermore, despite the small sample size, when the evidence presented in this study is considered together with previous findings in PM carriers ${ }^{37}$ and our earlier report of a significant association between CYC1 and FMR1 expression in GZ carriers with neurodevelopmental disorders, ${ }^{21}$ it suggests that the proposed link between elevated transcription at the FMR1 locus, CGG expansion within GZ/PM range, and mitochondrial dysfunction may extend beyond neurodegenerative disorders.

\section{ACKNOWLEDGMENTS}

This study was supported by the National Institutes of Child Health and Human Development Grant HD 36071, Australian National Health and Medical Research Council (NHMRC) Project Grant No. 330400 (to Dr. D. Z. Loesch), and the Principal Research Fellowship (Grant No. 436906) from the NHMRC (to Dr. D. R. Thorburn). The authors thank Dr. D. Amor from the Victorian Clinical Genetic Services for providing blood sample from one premutation carrier included in Figure 2, and Louise Hills and Con Ngo from the VCGS Cytogenetics Laboratory, Murdoch Childrens' Research Institute, for acquisition of patients' CGG expansion size data. They also thank the clinical patients and their families who participated in this study.

\section{REFERENCES}

1. Hagerman RJ, Leehey M, Heinrichs W, et al. Intention tremor, parkinsonism, and generalized brain atrophy in male carriers of fragile X. Neurology 2001;57:127-130.

2. Verkerk AJ, Pieretti M, Sutcliffe JS, et al. Identification of a gene (FMR-1) containing a CGG repeat coincident with a breakpoint cluster region exhibiting length variation in fragile X syndrome. Cell 1991;65:905-914.

3. Maddalena A, Richards CS, McGinniss MJ, et al. Technical standards and guidelines for fragile $\mathrm{X}$ : the first of a series of disease-specific supplements to the Standards and Guidelines for Clinical Genetics Laboratories of the American College of Medical Genetics. Quality Assurance Subcommittee of the Laboratory Practice Committee. Genet Med 2001;3:200-205.

4. Jacquemont S, Hagerman RJ, Leehey MA, et al. Penetrance of the fragile $\mathrm{X}$-associated tremor/ataxia syndrome in a premutation carrier population. JAMA 2004;291:460-469.

5. Jacquemont $\mathrm{S}$, Hagerman RJ, Leehey $\mathrm{M}$, et al. Fragile $\mathrm{X}$ premutation tremor/ataxia syndrome: molecular, clinical, and neuroimaging correlates. Am J Hum Genet 2003;72:869-878.

6. Loesch DZ, Churchyard A, Brotchie P, Marot M, Tassone F. Evidence for, and a spectrum of, neurological involvement in carriers of the fragile $\mathrm{X}$ pre-mutation: FXTAS and beyond. Clin Genet 2005;67:412-417.

7. Brunberg JA, Jacquemont S, Hagerman RJ, et al. Fragile X premutation carriers: characteristic MR imaging findings of adult male patients with progressive cerebellar and cognitive dysfunction. AJNR Am J Neuroradiol 2002;23:1757-1766.

8. Greco CM, Hagerman RJ, Tassone F, et al. Neuronal intranuclear inclusions in a new cerebellar tremor/ataxia syndrome among fragile $\mathrm{X}$ carriers. Brain 2002; 125:1760-1771

9. Song FJ, Barton P, Sleightholme V, Yao GL, Fry-Smith A. Screening for fragile X syndrome: a literature review and modelling study. Health Technol Assess 2003;7:1-106.

10. Tan EK, Zhao Y, Puong KY, et al. Fragile X premutation alleles in SCA, ET, and parkinsonism in an Asian cohort. Neurology 2004;63:362-363.

11. Deng H, Le W, Jankovic J. Premutation alleles associated with Parkinson disease and essential tremor. JAMA 2004;292:1685-1686.

12. Toft M, Aasly J, Bisceglio G, et al. Parkinsonism, FXTAS, and FMR1 premutations. Mov Disord 2005;20:230-233.

13. Kurz MW, Schlitter AM, Klenk Y, et al. FMR1 alleles in Parkinson's disease: relation to cognitive decline and hallucinations, a longitudinal study. $J$ Geriatr Psychiatry Neurol 2007;20:89-92.

14. Kraff J, Tang HT, Cilia R, et al. Screen for excess FMR1 premutation alleles among males with parkinsonism. Arch Neurol 2007;64:1002-1006.

15. Tassone F, Hagerman RJ, Taylor AK, Gane LW, Godfrey TE, Hagerman PJ. Elevated levels of FMR1 mRNA in carrier males: a new mechanism of involvement in the fragile-X syndrome. Am J Hum Genet 2000;66:6-15.

16. Loesch DZ, Bui QM, Huggins RM, Mitchell RJ, Hagerman RJ, Tassone F. Transcript levels of the intermediate size or grey zone fragile $\mathrm{X}$ mental retardation 1 alleles are raised, and correlate with the number of CGG repeats. J Med Genet 2007;44:200-204.

17. Loesch DZ, Litewka L, Churchyard A, Gould E, Tassone F, Cook M. Tremor/ataxia syndrome and fragile $\mathrm{X}$ premutation: diagnostic caveats. J Clin Neurosci 2007;14:245-248.

18. Loesch DZ, Khaniani MS, Slater HR, et al. Small CGG repeat expansion alleles of FMR1 gene are associated with parkinsonism. Clin Genet 2009; 76:471-476.

19. Li R, Luciakova K, Nelson BD. Expression of the human cytochrome c1 gene is controlled through multiple Sp1-binding sites and an initiator region. Eur J Biochem 1996;241:649-656.

20. Ladd PD, Smith LE, Rabaia NA, et al. An antisense transcript spanning the CGG repeat region of FMR1 is upregulated in premutation carriers but silenced in full mutation individuals. Hum Mol Genet 2007;16:3174-3187.

21. Loesch DZ, Godler DE, Khaniani M, et al. Linking the FMR1 alleles with small CGG expansions with neurodevelopmental disorders: preliminary data suggest an involvement of epigenetic mechanisms. Am J Med Genet A 2009;149A:2306-2310.

22. Khaniani MS, Kalitsis P, Burgess T, Slater HR. An improved Diagnostic PCR assay for identification of cryptic heterozygosity for CGG triplet repeat alleles in the fragile X gene (FMR1). Mol Cytogenet 2008;1:5.

23. Pagnamenta AT, Taanman JW, Wilson CJ, et al. Dominant inheritance of premature ovarian failure associated with mutant mitochondrial DNA polymerase gamma. Hum Reprod 2006;21:2467-2473.

24. Bustin SA. Quantification of mRNA using real-time reverse transcription PCR (RT-PCR): trends and problems. J Mol Endocrinol 2002;29:23-39.

25. Godler DE, Loesch DZ, Huggins R, et al. Improved methodology for assessment of mRNA levels in blood of patients with FMR1 related disorders. BMC Clin Pathol 2009;9:5.

26. Pietrobono R, Tabolacci E, Zalfa F, et al. Molecular dissection of the events leading to inactivation of the FMR1 gene. Hum Mol Genet 2005;14:267277.

27. Khalil AM, Faghihi MA, Modarresi F, Brothers SP, Wahlestedt C. A novel 
RNA transcript with antiapoptotic function is silenced in fragile $\mathrm{X}$ syndrome. PLoS One 2008;3:e1486.

28. Fahn S, Eltan RL, and Members of the UPDRS Development Committee. Recent developments in Parkinson's disease. Unified Parkinson's Disease Rating Scale. Florham Park, NJ: Macmillan Health Care Information;1987: $153-164$.

29. Richards M, Marder K, Cote L, Mayeux R. Interrater reliability of the Unified Parkinson's Disease Rating Scale motor examination. Mov Disord 1994;9:89-91.

30. Despres C, Lamoureux D, Beuter A. Standardization of a neuromotor test battery: the CATSYS system. Neurotoxicology 2000;21:725-735.

31. Letz R, Gerr F. Reliability of some tremor measurement outcome variables in field testing situations. Neurotoxicology 2000;21:737-742.

32. Mathuranath PS, Nestor PJ, Berrios GE, Rakowicz W, Hodges JR. A brief cognitive test battery to differentiate Alzheimer's disease and frontotemporal dementia. Neurology 2000;55:1613-1620.

33. Kukull WA, Larson EB, Teri L, Bowen J, McCormick W, Pfanschmidt ML. The Mini-Mental State Examination score and the clinical diagnosis of dementia. J Clin Epidemiol 1994;54:1050-1058.

34. Smith A. Symbol digit modalities test. Los Angeles, CA: Western Psychological Services, 1991.

35. R Foundation for statistical computing [computer program]. A language and environment for statistical computing. Version 1.191. Vienna, Austria: R Foundation for statistical computing, 2009.

36. Vandesompele J, De Preter K, Pattyn F, et al. Accurate normalization of real-time quantitative RT-PCR data by geometric averaging of multiple internal control genes. Genome Biol 2002;3:RESEARCH0034.

37. Ross-Inta C, Omanska-Klusek A, Wong S, et al. Evidence of mitochondrial dysfunction in fragile $\mathrm{X}$-associated tremor/ataxia syndrome. Biochem $\mathrm{J}$ 2010;429:545-552.

38. Sellem CH, Marsy S, Boivin A, Lemaire C, Sainsard-Chanet A. A mutation in the gene encoding cytochrome $\mathrm{cl}$ leads to a decreased ROS content and to a long-lived phenotype in the filamentous fungus Podospora anserina. Fungal Genet Biol 2007;44:648-658.

39. Acin-Perez R, Bayona-Bafaluy MP, Fernandez-Silva P, et al. Respiratory complex III is required to maintain complex I in mammalian mitochondria. Mol Cell 2004;13:805-815.

40. Schapira AH. Mitochondria in the aetiology and pathogenesis of Parkinson's disease. Lancet Neurol 2008;7:97-109.

41. Benmoyal-Segal L, Soreq H. Gene-environment interactions in sporadic Parkinson's disease. J Neurochem 2006;97:1740-1755.

42. Haas RH, Nasirian F, Nakano K, et al. Low platelet mitochondrial complex I and complex II/III activity in early untreated Parkinson's disease. Ann Neurol 1995;37:714-722.

43. Silva JM, Wong A, Carelli V, Cortopassi GA. Inhibition of mitochondrial function induces an integrated stress response in oligodendroglia. Neurobiol Dis 2009;34:357-365.

44. Mineri R, Pavelka N, Fernandez-Vizarra E, Ricciardi-Castagnoli P, Zeviani M, Tiranti V. How do human cells react to the absence of mitochondrial DNA? PLoS One 2009;4:e5713.

45. Rizzo G, Pizza F, Scaglione C, et al. A case of fragile X premutation tremor/ataxia syndrome with evidence of mitochondrial dysfunction. Mov Disord 2006;21:1541-1542.

46. Henchcliffe C, Shungu DC, Mao X, et al. Multinuclear magnetic resonance spectroscopy for in vivo assessment of mitochondrial dysfunction in Parkinson's disease. Ann N Y Acad Sci 2008;1147:206-220.

47. Galvao R, Mendes-Soares L, Camara J, Jaco I, Carmo-Fonseca M. Triplet repeats, RNA secondary structure and toxic gain-of-function models for pathogenesis. Brain Res Bull 2001;56:191-201.

48. Bueler H. Impaired mitochondrial dynamics and function in the pathogenesis of Parkinson's disease. Exp Neurol 2009;218:235-246. 